\title{
Low-symmetrical Zinc(II) Benzonaphthoporphyrazine Sensitizers for Light-Harvesting in Near-IR Region of Dye-Sensitized Solar Cells
}

Takuro Ikeuchi, Shogo Mori, Nagao Kobayashi, and Mutsumi Kimura*

Division of Chemistry and Materials, Faculty of Textile Science and Technology, Shinshu University, Ueda 386-8567, Japan

\section{Supporting Information}

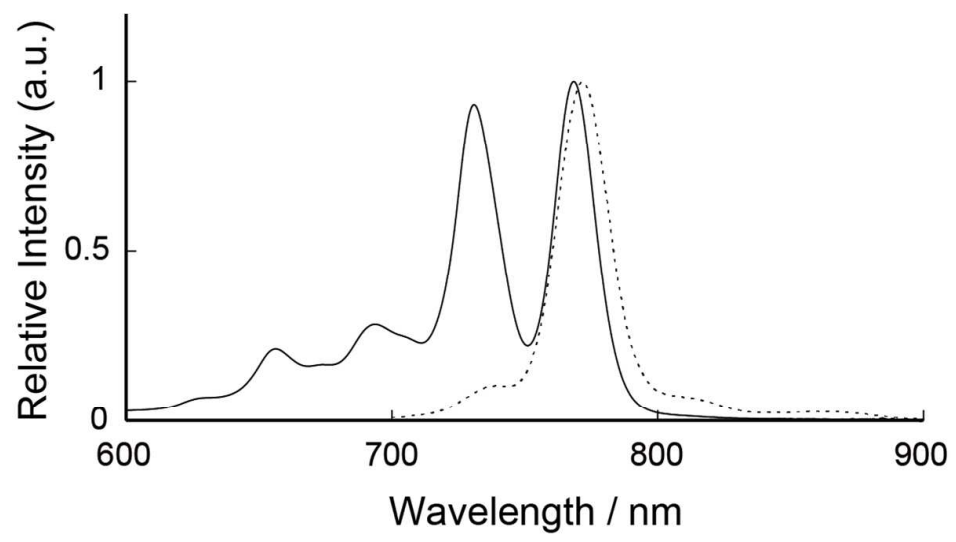

Figure S1. Absorption (solid line) and fluorescence (dotted line) spectra of NcS1 in toluene.

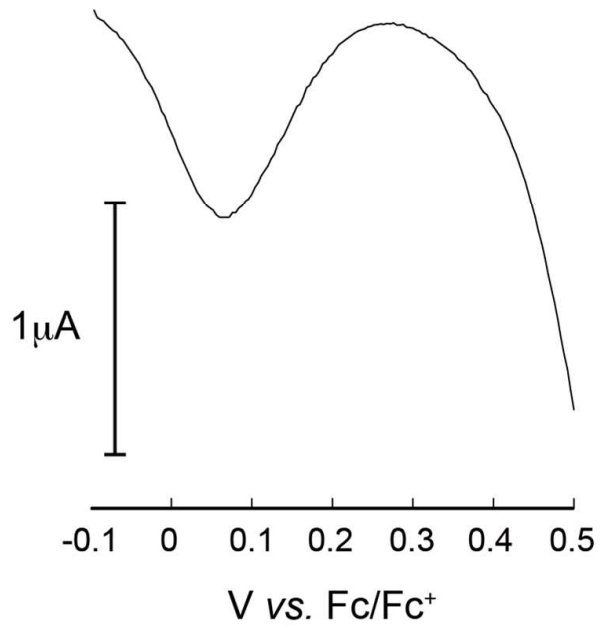

Figure S2. Differential pulse voltammogram of $\mathbf{N c S 1}$ adsorbed onto $\mathrm{TiO}_{2}$ nanopartiles obtained in acetonitrile containing $0.1 \mathrm{M} \mathrm{TBAPF}_{6}$ 


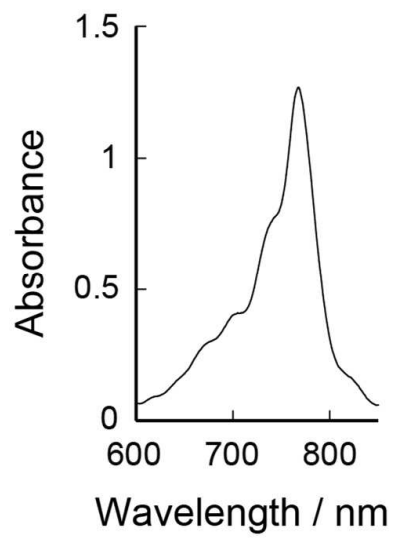

Figure S3. Absorption spectrum of dye-stained $\mathrm{TiO}_{2}$ film of $\mathbf{N c S 1}+\mathrm{CDCA}$.
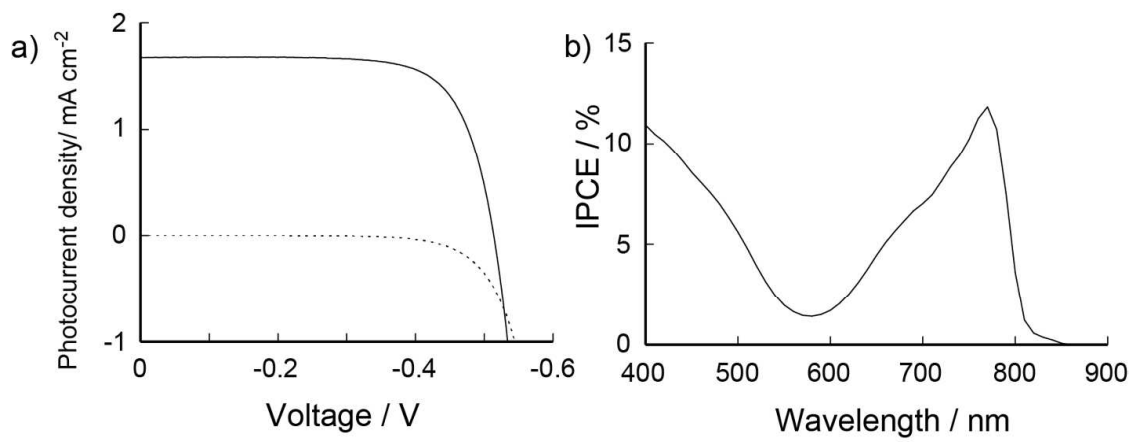

Figure S4. a) Photocurrent voltage curves obtained with DSSCs based on NcS2 under a standard global AM 1.5 solar condition (solid line) and dark current (dotted line). b) Incident photon-to-current conversion efficiency spectrum for DSSC based on NeS2. 